\title{
VARIABILIDADE TEMPORAL DA PRECIPITAÇÃO MENSAL E ANUAL NA ESTAÇÃO CLIMATOLÓGICA DE UBERABA-MG
}

\begin{abstract}
RESUMO - A análise da variabilidade espacial e da variabilidade temporal de atributos naturais vem recebendo destaque nos últimos tempos, devido à possibilidade de se realizar estimativas com maior precisão, destacando-se entre esses atributos a precipitação pluviométrica. Objetivou-se com este trabalho estudar o comportamento temporal das chuvas mensais na Estação Climatológica de Uberaba - MG, utilizando-se a série das precipitações pluviais mensais e anuais de 1914 a 2000. Os dados referentes à Estação Climatológica de Uberaba - Estação Experimental Getúlio Vargas - foram obtidos no Laboratório de Climatologia e Recursos
\end{abstract}

\author{
JOSÉ WALDEMAR DA SILVA ${ }^{1}$ \\ EDNALDO CARVALHO GUIMARÃES ${ }^{2}$ \\ MARCELO TAVARES ${ }^{3}$
}

\begin{abstract}
Hídricos do Instituto de Geografia - Universidade Federal de Uberlândia. Realizou-se análise exploratória dos dados por meio de algumas estatísticas, como média aritmética, desvio-padrão, valores máximos e valores mínimos e histogramas. A análise de variabilidade temporal foi feita por meio de semivariâncias. Verificou-se uma tendência à normalidade nos meses correspondentes ao perído de outubro a março e assimetria no período de abril a setembro. Observou-se a presença do efeito pepita puro, não constatando-se dependência temporal e, conseqüientemente, estudos e inferências estatísticas podem ser realizados com base em métodos da estatística não espacial.
\end{abstract}

TERMOS PARA INDEXAÇÃO: Geoestatística, semivariograma, dependência temporal, precipitação.

\section{TEMPORAL VARIABILITY OF MONTHLY RAINS IN THE CLIMATOLOGICAL STATION OF UBERABA-MG, BRAZIL}

\begin{abstract}
The purpose of this work was to verify the temporal dependence of monthly rainfall in the climatological station of Uberaba-MG, Brazil, using geostatistical methods. It was evaluated the precipitation in series of rainfall monthly data from 1914 to 2000. It were done exploratory and geostatistical analysis. The exploratory analysis was made by calculating basic statistics and histograms and
\end{abstract}

the geostatistical analysis was made by semivariogram. According to the results, a normality tendence was verified in months from october to march. From april to September, it was verified an asymmetric tendence. Semivaviograms showed the nugget effect. As a conclusion, these variables did not present spatial dependence and statistical inferences can be made assuming temporal independence.

INDEX TERMS: Geostatistics, semivariogram, spatial dependence, rainfall.

\section{INTRODUÇÃO}

A precipitação pluviométrica tem grande importância na caracterização do clima de uma região, interferindo nas alternâncias de rendimento das culturas. O estudo dessa variável, de grande influência na caracterização do clima da região do Triângulo Mineiro, torna-se relevante no planejamento de atividades agríco- las, permitindo previsões com melhores aproximações e decisões mais confiáveis.

Aplicações de modelos matemáticos e de modelos estatísticos têm explicado o comportamento dos fenômenos que ocorrem na natureza, em particular na agricultura.

Dentre as técnicas estatísticas usadas para análise e interpretação de dados climáticos, encontra-se a Geoestatística, que tem como característica principal a

1. Acadêmico do Curso de Matemática - FAMAT/UFU - jwsilva@alunos.ufu.br - Bolsista PIBIC/CNPq.

2. Professor, Dr. - Orientador - FAMAT/UFU - Av. João Naves de Ávila, no 2160 - Uberlândia, MG - 38400-089 ecg@ufu.br

3. Professor, Dr. -FAMAT/UFU - mtavares@ufu.br 
análise da distribuição espacial e/ou temporal entre as observações, determinando, por meio do semivariograma, a distância ou período de tempo de dependência entre elas. Em Freitas (2000), encontra-se uma descrição sobre a análise espacial de dados utilizando semivariogramas.

Vieira et al. (1991) estudaram o mapeamento das chuvas diária máxima provável para o Estado de São Paulo e utilizaram a autocorrelação espacial, calculada pelo semivariograma, para verificar a dependência espacial e realizar estimativas para os locais onde não se têm valores medidos.

Estudando o comportamento espacial do percentil 75 da precipitação decendial do Estado de São Paulo, Gomes (2001) elaborou mapas de isoeitas utilizando como ferramenta principal a Geoestatística.

Cardim (2001) realizou o mapeamento multivariado das principais variáveis climáticas de interesse agrícola do Estado de São Paulo e a Geoestatística permitiu analisar a variabilidade espacial dos índices climáticos obtidos (índice térmico, índice pluviométrico e índice pluviotérmico).

Para o Estado do Paraná, Zamboti (2001) realizou o mapeamento de chuvas médias mensais nos meses de verão, utilizando como ferramenta de análise da dependência espacial e interpolação de dados, respectivamente, o semivariograma e a krigagem.

A amostragem simples ao acaso nem sempre é a forma mais eficiente de estimar parâmetros relacionados a fenômenos naturais, visto que, na maioria dos casos, não se dispõe de condições ideais, ou seja, não se consegue satisfazer a hipótese de independência entre as amostras.

O estudo da variabilidade temporal da precipitação permite definir o grau de correlação temporal das amostras e tem mostrado ser poderosa ferramenta de aplicação prática, permitindo estimar precipitações com variância mínima.

A análise espaço-temporal da precipitação tem grande aplicabilidade na construção civil e principalmente na agricultura e apresenta uma literatura ainda carente e pouco explorada, mas, certamente, é um assunto que se tornará de grande importância em produções científicas.

Com este trabalho objetivou-se estudar a distribuição temporal da precipitação pluviométrica na Estação Climatológica de Uberaba-MG, utilizando o semivariograma como ferramenta de determinação da dependência temporal.

\section{MATERIAL E MÉTODOS}

Os dados de precipitação pluviométrica, utilizados no presente estudo, são provenientes da Estação Experimental Getúlio Vargas, da Empresa de Pesquisa Agropecuária de Minas Gerais (EPAMIG), localizada no município de Uberaba, Estado de Minas Gerais, em convênio com o Instituto Nacional de Meteorologia (INMET).

A Estação Climatológica de Uberaba encontrase na latitude de $19^{\circ} 59^{\prime} \mathrm{S}$, longitude de $47^{\circ} 53^{\prime} \mathrm{W}$ e altitude de 742,9 metros. Segundo a classificação internacional de Köppen, o clima da região é do tipo Aw, isto é, tropical quente úmido, com inverno frio e seco (Terra, 2002). O total médio de chuvas no mês mais seco da série foi de $12,5 \mathrm{~mm}$ (mês de agosto) e no mês mais chuvoso foi de $283,6 \mathrm{~mm}$ (mês de janeiro) e o total anual médio, de $1584,2 \mathrm{~mm}$.

Os dados utilizados foram obtidos no Laboratório de Climatologia e Recursos Hídricos do Instituto de Geografia da Universidade Federal de Uberlândia, abrangendo o período de 1914 a 2000. As observações referem-se às precipitações pluviais mensais e anuais expressas em altura de lâmina d'água $(\mathrm{mm})$.

Realizou-se a análise exploratória das variáveis precipitações mensais e precipitações anuais, sendo obtidas, para cada mês do ano, as estatísticas: média aritmética $(\bar{X})$, mediana (Md), variância $\left(\mathrm{s}^{2}\right)$, desviopadrão (s), valor mínimo (Min), valor máximo (Max), limite inferior e superior do intervalo de confiança de 95\% para a média; e, ainda, fez-se a representação gráfica, por meio de histogramas, para verificar sua adequação à distribuição normal. A análise exploratória teve a finalidade de caracterizar a distribuição de probabilidade e verificar a variabilidade dos atributos em estudo.

A análise Geoestatística foi utilizada com o objetivo de definir o modelo de variabilidade temporal do total mensal e do total anual de chuva na Estação Experimental Getúlio Vargas. A análise variográfica foi feita por meio de semivariogramas, que mostram uma série de pontos discretos de $\gamma(\mathrm{t})$ correspondendo a cada valor de $\mathrm{t}$ (semivariância experimental), e para o qual uma função contínua (modelo teórico) deve ser ajustada, conforme mostra Vieira (1995).

Gráficos da semivariância $(\gamma)$ em função do tempo (t) foram utilizados na definição dos semivariogramas referentes a cada mês do ano. $\mathrm{O}$ cálculo das semivariâncias e a escolha do modelo de semivario-

Ciênc. agrotec., Lavras. V.27, n.3, p.665-674, maio/jun., 2003 
grama foram realizados pelo programa VARIOWIN 2.2, desenvolvido por Pannatier (1996).

As semivariâncias foram calculadas pela equação:

$$
\gamma(h)=\frac{1}{2 N(h)} \sum_{i=1}^{N(h)}[Z(x)-Z(x+h)]^{2}
$$

em que:

$\gamma(\mathrm{h})$ é a semivariância para um vetor h (anos);

$\mathrm{Z}(\mathrm{x})$ e $\mathrm{Z}(\mathrm{x}+\mathrm{h})$ são os pares de observações de precipitação pluviométrica, separados pelo vetor $\mathrm{h}$ (anos);

$\mathrm{N}(\mathrm{h})$ é o número de pares de valores medidos $\mathrm{Z}(\mathrm{x})$, $\mathrm{Z}(\mathrm{x}+\mathrm{h})$, separados por um vetor $\mathrm{h}$.

\section{RESULTADOS E DISCUSSÃO}

As estatísticas das variáveis precipitação mensal e precipitação anual são apresentadas no Quadro 1.

Observa-se que o número de registros de precipitação pluviométrica é de 62 , ou seja, tem-se informação de apenas 62 anos da série estudada. As falhas de registros foram observadas com maior freqüência nos pri- meiros anos de obtenção dos dados. Aspiazú et al. 1990), Campelo Júnior (1993) e Goovaerts (2000) argumentam que a falta de registros contínuos é um dos fatores que pode dificultar a elaboração de classificações climáticas precisas.

Os valores médios de precipitação mensal revelam uma estação seca que corresponde aos meses de maio a setembro e uma estação chuvosa que corresponde aos meses de outubro a abril.

Os coeficientes de variação da precipitação mensal (Quadro 1) mostram que a variabilidade desse atributo é relativamente alta, de acordo com a classificação de Gomes (2000). A variabilidade se torna mais pronunciada na estação seca, que apresenta coeficientes de variação entre $61,37 \%$ e $161,08 \%$. A ausência total de chuvas em alguns anos da série, no período da seca, pode ser a explicação dessa variabilidade e, esse fato indica que estimativas feitas com a média aritmética podem apresentar precisão e confiabilidade duvidosas, pelo fato de essa medida de posição não ser a mais adequada para representar a variável, pois é altamente influenciada por valores extremos.

QUADRO 1 - Estatísticas da precipitação total mensal (mm) e total anual (mm) na Estação Climatológica de Uberaba - MG, no período de 1914 a 2000.

\begin{tabular}{cccccccccc}
\hline Mês & $\mathbf{n}$ & $\bar{X}$ & $\mathbf{s}$ & $\mathbf{C V}$ & $\mathbf{L I}(\mathbf{9 5 \%})$ & $\mathbf{L S}(\mathbf{9 5 \%})$ & Md & Min & Max \\
\hline Jan & 62 & 283,6 & 92,1 & 32,5 & 260,3 & 307,1 & 277,9 & 118,8 & 541 \\
Fev & 62 & 221,2 & 85,9 & 38,8 & 199,4 & 243,0 & 213,2 & 42,8 & 453,2 \\
Mar & 62 & 199,9 & 96,2 & 48,1 & 175,5 & 224,3 & 197,5 & 28 & 616 \\
Abr & 62 & 101,7 & 62,4 & 61,3 & 85,9 & 117,6 & 92,5 & 0 & 268,1 \\
Mai & 62 & 45,2 & 39,7 & 87,7 & 35,2 & 55,3 & 40,6 & 0 & 177,7 \\
Jun & 62 & 20,2 & 23,0 & 114,0 & 14,3 & 26,0 & 11,0 & 0 & 100,6 \\
Jul & 62 & 13,7 & 21,2 & 153,9 & 8,4 & 19,1 & 0,4 & 0 & 83,6 \\
Ago & 62 & 12,5 & 20,2 & 161,0 & 7,4 & 17,6 & 2,8 & 0 & 78,4 \\
Set & 62 & 62,3 & 54,6 & 87,7 & 48,4 & 76,1 & 47,3 & 0 & 242,7 \\
Out & 62 & 140,5 & 72,0 & 51,2 & 122,2 & 158,8 & 137,1 & 0 & 410 \\
Nov & 62 & 201,7 & 79,0 & 39,1 & 181,7 & 221,8 & 192,8 & 54,3 & 438,9 \\
Dez & 62 & 281,1 & 82,0 & 29,1 & 260,3 & 301,9 & 289,8 & 119,3 & 438,5 \\
\hline Anual & 62 & 1584,2 & 229,0 & 14,4 & 1526,0 & 1642,4 & 1575,8 & 1193,2 & 2158,6 \\
\hline
\end{tabular}

n- Amostra; $\bar{X}$-Média; s - Desvio-Padrão; CV - Coeficiente de Variação; LI(95\%) - Limite Inferior do Intervalo de Confiança para média com $95 \%$ de confiança; LS(95\%) - Limite Superior do Intervalo de Confiança para média com $95 \%$ de confiança; Md - Mediana; MIN - Valor Mínimo; MAX - Valor Máximo. 
Os limites inferiores e superiores do intervalo de confiança de $95 \%$ para a média mensal revelam diferenças significativas entre a precipitação nas duas estações, não ocorrendo interseção dos intervalos para os meses de abril a setembro e outubro a março.

Os valores da mediana que se afastam em relação aos valores da média aritmética e também os valores mínimos e máximos reforçam a observação de alta variabilidade do atributo em estudo.

A alta variabilidade de dados de precipitação pluviométrica e a baixa confiabilidade na média aritmética para representar essa variável também foram verificadas por Medina \& Leite (1984), Eltz et al. (1992) e Ribeiro \& Lunardi (1997).

$\mathrm{O}$ atributo precipitação anual mostrou-se relativamente pouco variável $(\mathrm{CV}=14 \%)$. Esse fato ocorre por esse valor ser a somatória de toda a chuva do ano e, conseqüentemente, meses relativamente secos em determinados anos são compensados por precipitações de meses chuvosos e, no total, os valores finais de ano para ano são mais uniformes do que dentro dos anos.

Nas Figuras 1 a 13 encontra-se o comportamento da variável precipitação mensal e anual na série estudada.
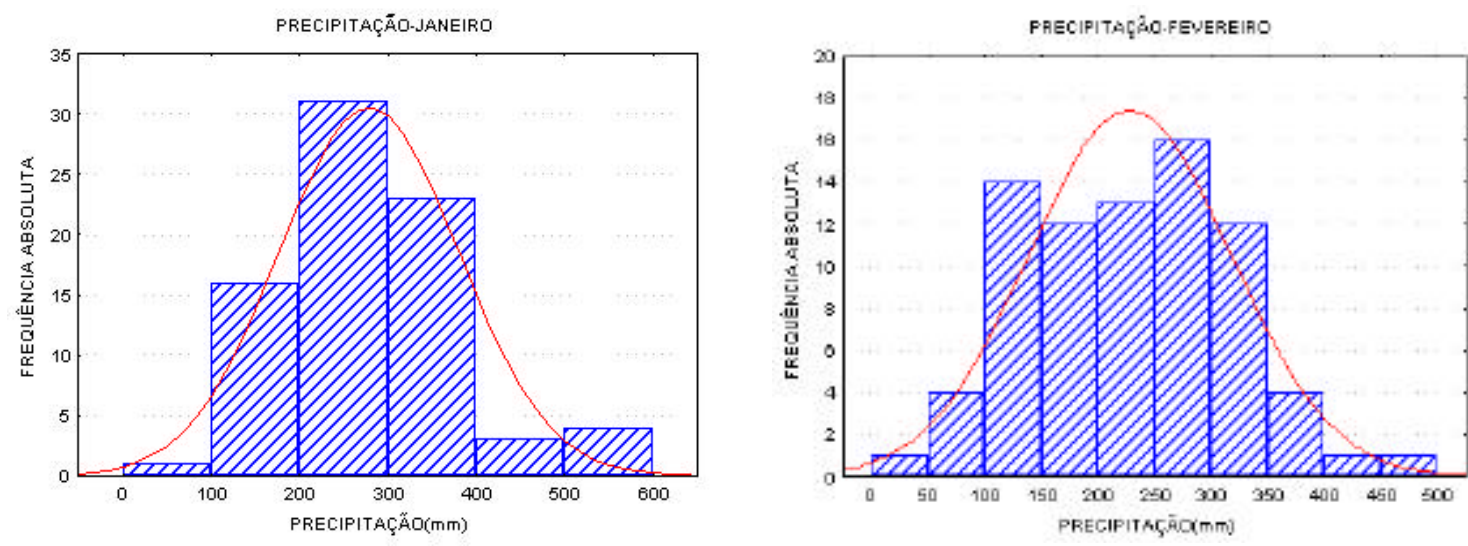

FIGURA 1 - Histograma da precipitação $(\mathrm{mm})$, do FIGURA 2 - Histograma da precipitação (mm), do mês de janeiro, na Estação Experimental Getúlio Var- mês de fevereiro, na Estação Experimental Getúlio gas - Uberaba - MG, no período de 1914 a 2000. Vargas - Uberaba - MG, no período de 1914 a 2000.
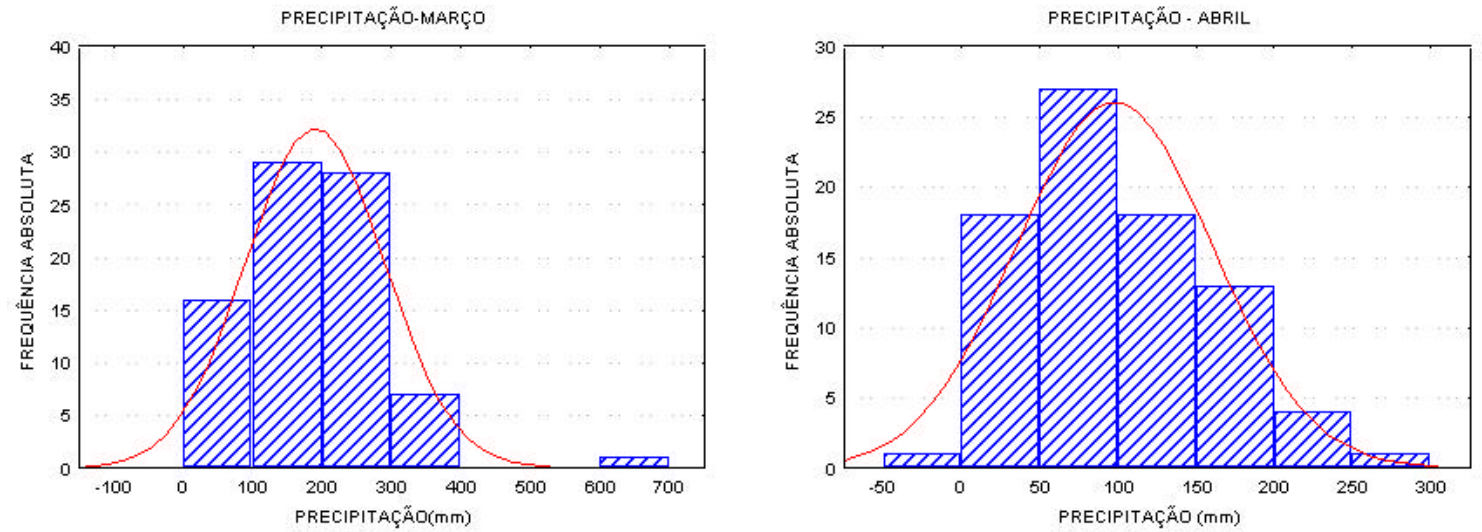

FIGURA 3 - Histograma da precipitação (mm), do FIGURA 4 - Histograma da precipitação (mm), do mês de março, na Estação Experimental Getúlio Var- mês de abril, na Estação Experimental Getúlio Vargas - Uberaba - MG, no período de 1914 a 2000. gas - Uberaba - MG, no período de 1914 a 2000

Ciênc. agrotec., Lavras. V.27, n.3, p.665-674, maio/jun., 2003 

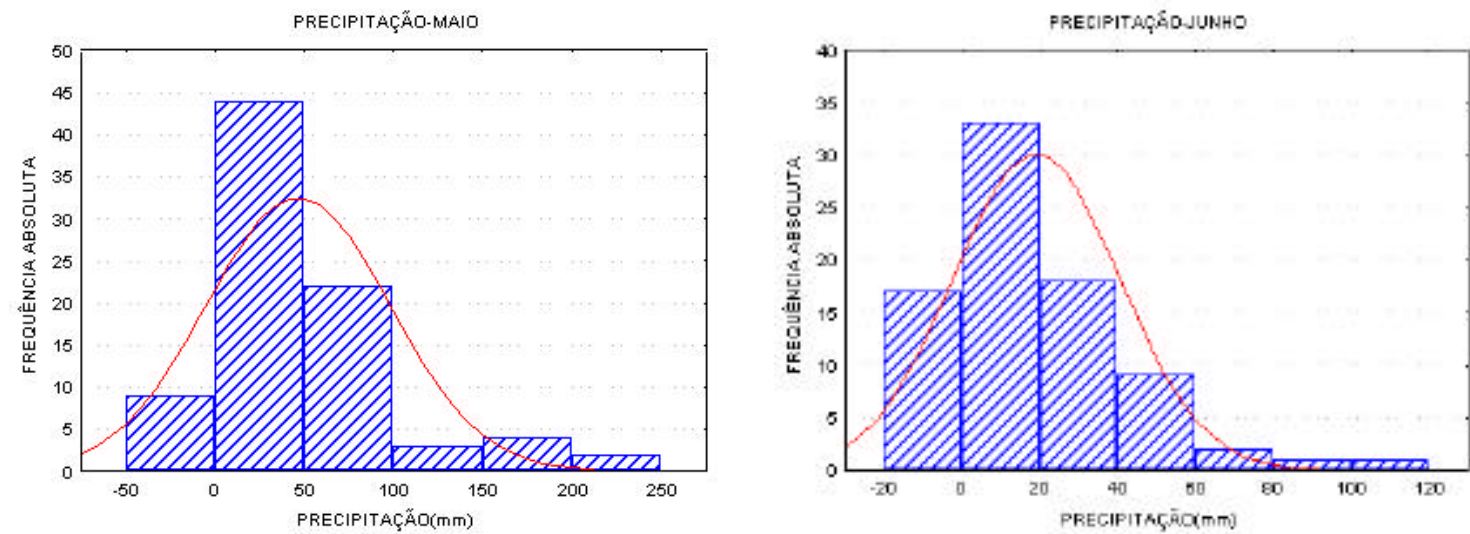

FIGURA 5 - Histograma da precipitação (mm), do FIGURA 6 - Histograma da precipitação (mm), do mês de maio, na Estação Experimental Getúlio Var- mês de junho, na Estação Experimental Getúlio Vargas - Uberaba - MG, no período de 1914 a 2000. gas - Uberaba - MG, no período de 1914 a 2000.
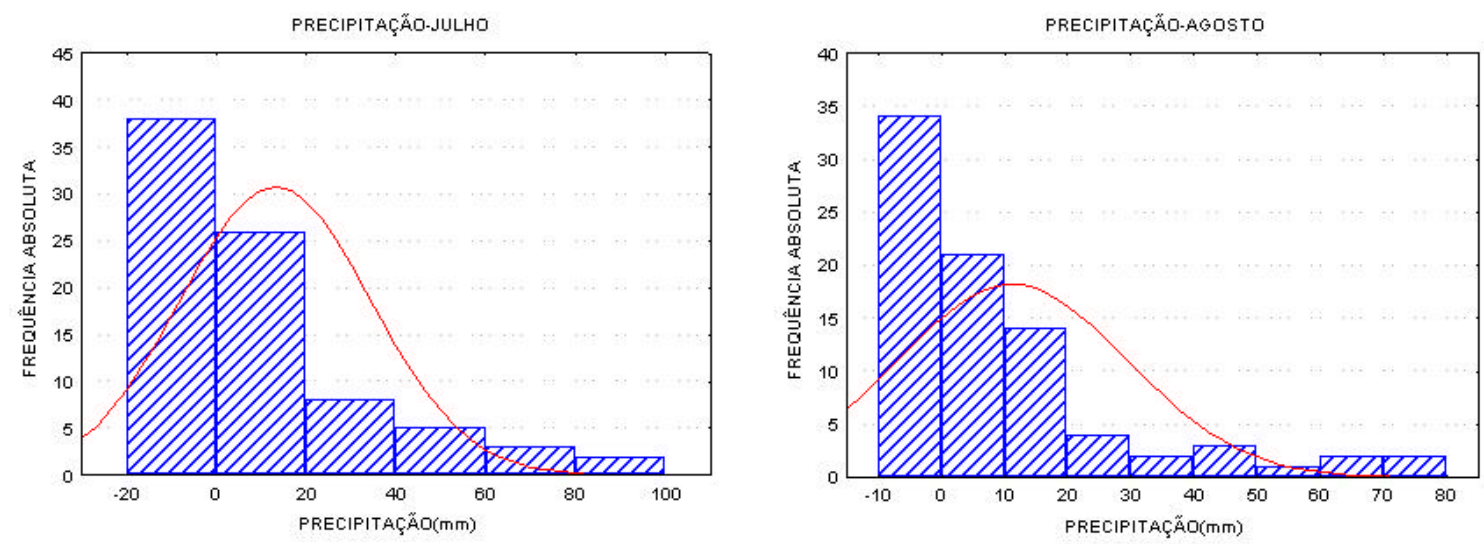

FIGURA 7 - Histograma da precipitação (mm), do FIGURA 8 - Histograma da precipitação (mm), do mês de julho, na Estação Experimental Getúlio Var- mês de agosto, na Estação Experimental Getúlio Vargas - Uberaba - MG, no período de 1914 a 2000. gas - Uberaba - MG, no período de 1914 a 2000.
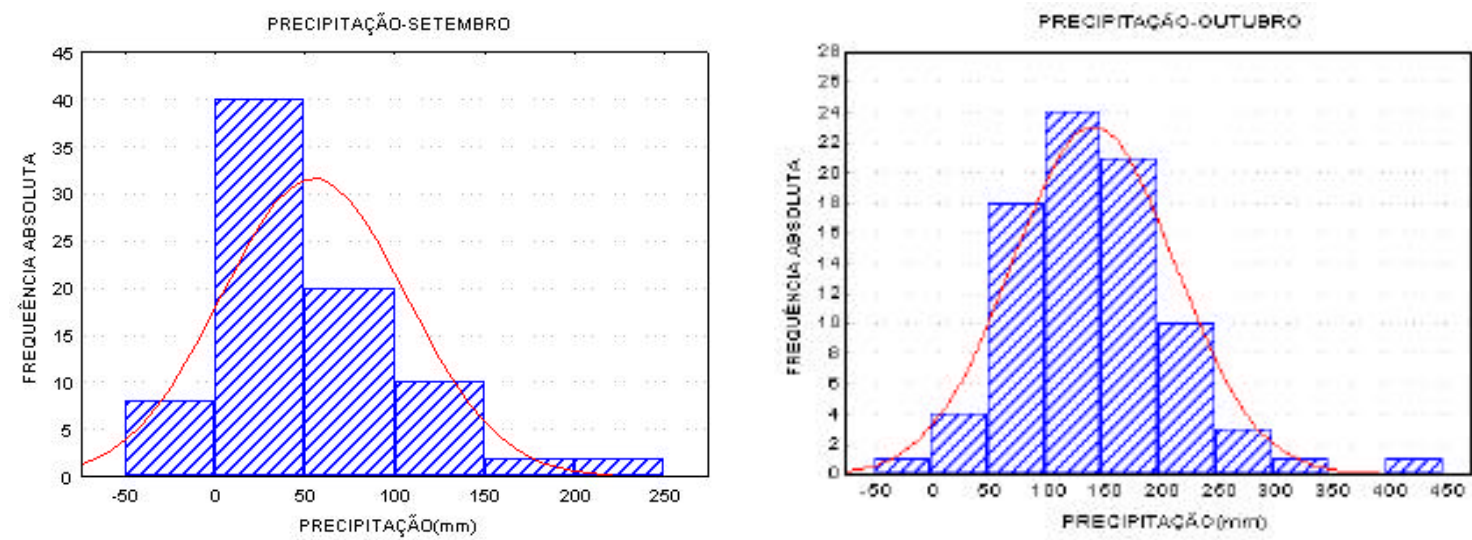

FIGURA 9 - Histograma da precipitação (mm), do FIGURA 10 - Histograma da precipitação (mm), do mês de setembro, na Estação Experimental Getúlio mês de outubro, na Estação Experimental Getúlio Vargas - Uberaba - MG, no período de 1914 a 2000. Vargas - Uberaba - MG, no período de 1914 a 2000. 

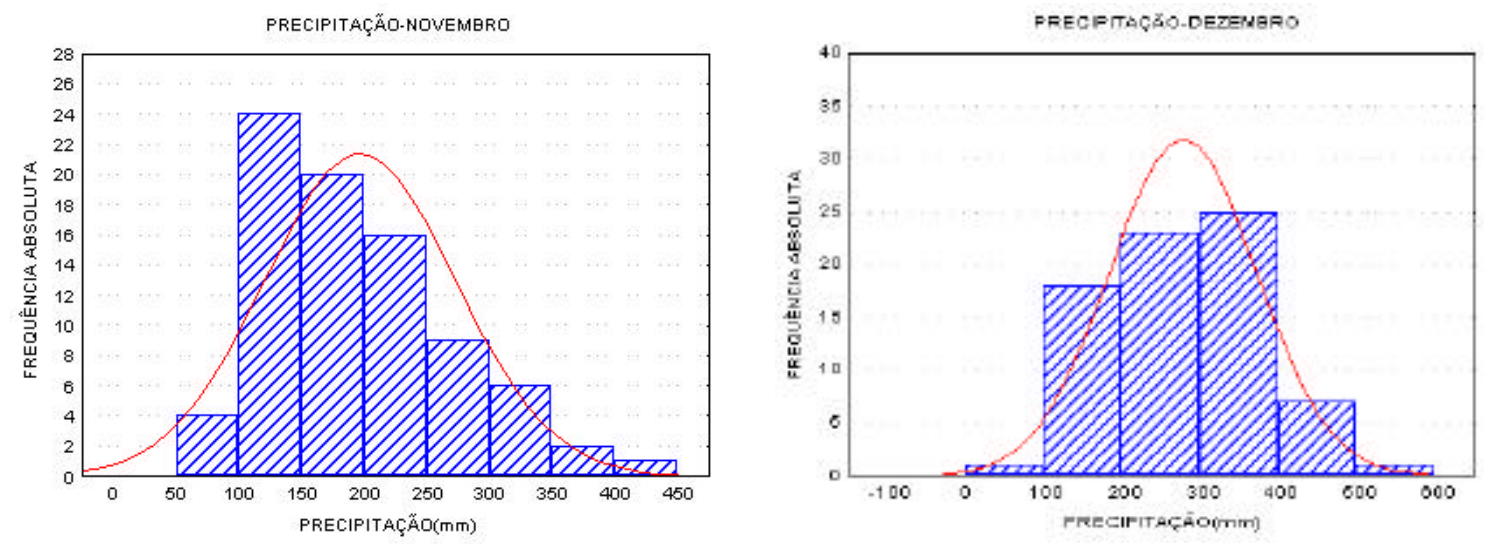

FIGURA 11 - Histograma da precipitação (mm), do FIGURA 12 - Histograma da precipitação (mm), do mês de novembro, na Estação Experimental Getúlio mês de dezembro, na Estação Experimental Getúlio Vargas - Uberaba - MG, no período de 1914 a 2000. Vargas - Uberaba - MG, de 1914 a 2000.

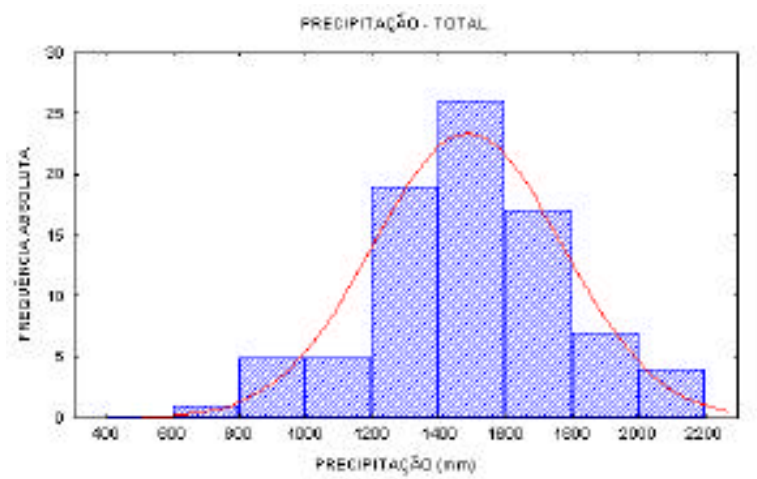

FIGURA 13 - Histograma da precipitação anual (mm), na Estação Experimental Getúlio Vargas - Uberaba - MG, no período de 1914 a 2000.

Observa-se assimetria acentuada para os meses de maio a setembro e tendência à simetria para os demais meses e também para os valores anuais.

A análise exploratória dos dados revelou que a precipitação mensal na Estação Climatológica de Uberaba se comporta de forma diferenciada entre os meses do ano, definindo claramente um período seco, com precipitação abaixo de $150 \mathrm{~mm} / \mathrm{mês}$, e um período chuvoso, com precipitação acima de $150 \mathrm{~mm} / \mathrm{mês}$.

Os semivariogramas experimentais são apresentados nas Figuras 14 a 26.

Verifica-se por meio das Figuras 14, 18, 21, 25 e 26, que representam, respectivamente, os semivariogramas para o meses de janeiro, maio, agosto, dezembro e do total anual, que ocorreu uma fraca dependência temporal da precipitação.

$\mathrm{O}$ ajuste de modelos de semivariogramas, com alcance da dependência temporal de no máximo 12 anos, associado à técnica de krigagem, pode ser utilizado para realizar estimativas da precipitação mensal em curtos períodos de tempo e, nesses casos, uma aplicação prática pode estar associada às estimativas das falhas existentes nos registros históricos, utilizando, como informações básicas, o modelo de dependência temporal e os registros de anos vizinhos em relação ao valor a ser estimado.

Observa-se também que os efeitos pepita dos semivariogramas representam valores superiores a $75 \%$ do patamar, que é classificado por Cambardella et al. (1994) como uma fraca dependência temporal. Nota-se, ainda, que, para o mês de agosto (Figura 21), o semivariograma é crescente para toda a série pesquisa. Semivariogramas com tendência crescente foram obtidos por Vieira (1997) e Guimarães (2000) em estudos sobre dependência espacial. Esses autores argumentam que tal fato pode estar relacionado a algum tipo de tendência da variável.

Os semivariogramas experimentais apresentados nas demais figuras revelam um comportamento aleatório para a precipitação pluviométrica, pois os semivariogramas apresentaram efeito pepita puro. Tal fato mostra que não ocorre dependência temporal das precipitações mensais e da precipitação anual e, portanto, métodos de estimação que consideram independência entre as amostras podem ser utilizados na avaliação e previsão temporal nesses casos. 


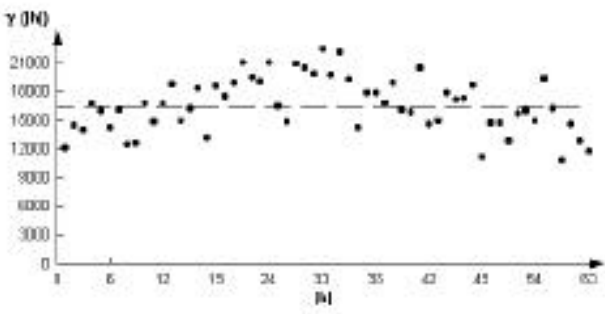

FIGURA 14 - Semivariograma experimental da precipitação (mm), do mês de janeiro, na Estação Experimental Getúlio Vargas - Uberaba - MG, no período de 1914 a 2000.

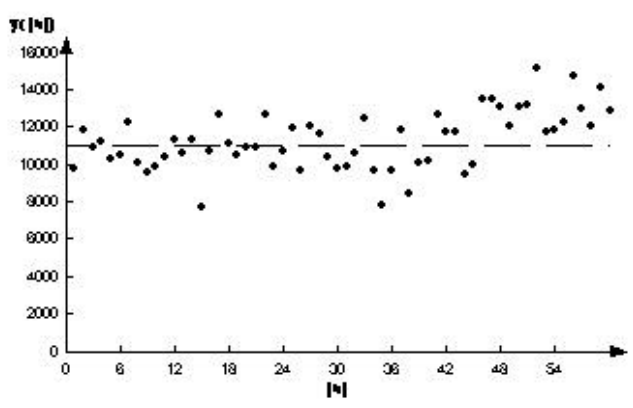

FIGURA 16 - Semivariograma experimental da precipitação (mm), do mês de março, na Estação Experimental Getúlio Vargas - Uberaba - MG, no período de 1914 a 2000.

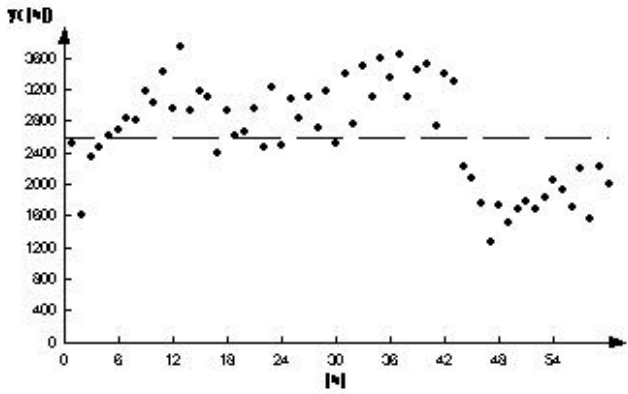

FIGURA 18 - Semivariograma experimental da precipitação (mm), do mês de maio, na Estação Experimental Getúlio Vargas - Uberaba - MG, no período de 1914 a 2000.

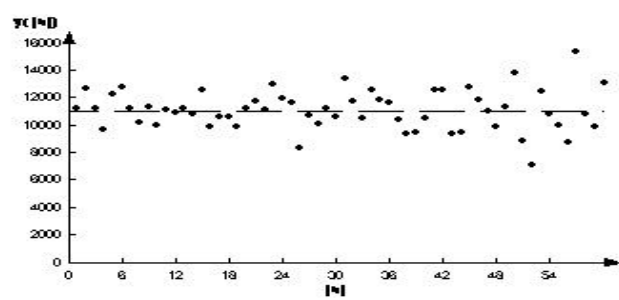

FIGURA 15 - Semivariograma experimental da precipitação (mm), do mês de fevereiro, na Estação Experimental Getúlio Vargas - Uberaba - MG, no período de 1914 a 2000.

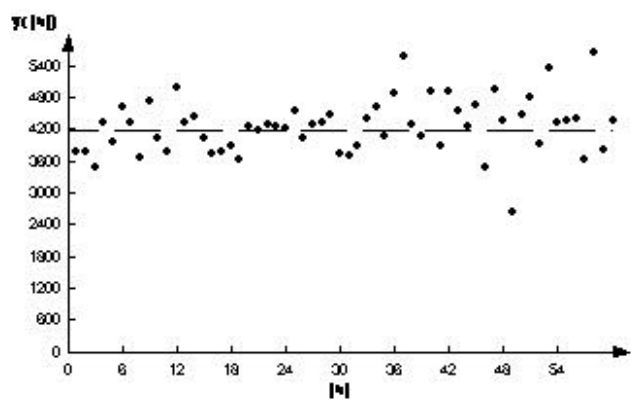

FIGURA 17 - Semivariograma experimental da precipitação (mm), do mês de abril, na Estação Experimental Getúlio Vargas - Uberaba - MG, no período de 1914 a 2000.

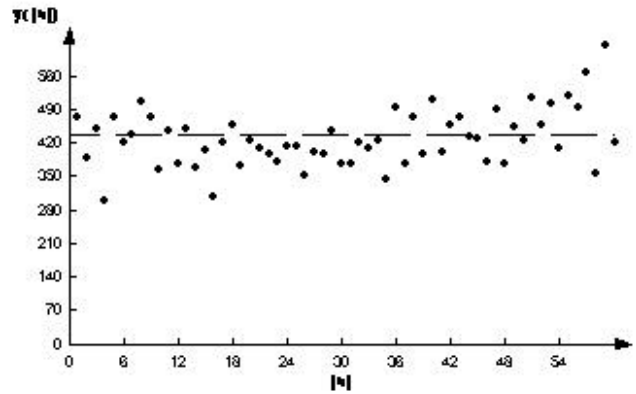

FIGURA 19 - Semivariograma experimental da precipitação (mm), do mês de junho, na Estação Experimental Getúlio Vargas - Uberaba - MG, no período de 1914 a 2000. 


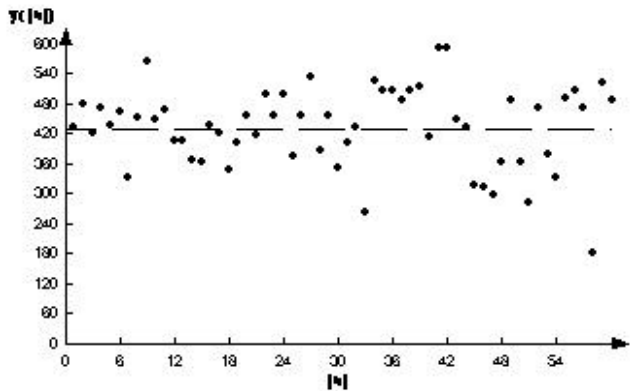

FIGURA 20 - Semivariograma experimental da precipitação (mm), do mês de julho, na Estação Experimental Getúlio Vargas - Uberaba - MG, no período de 1914 a 2000.

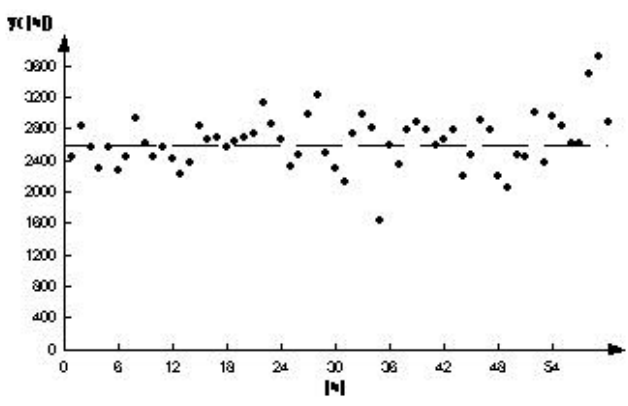

FIGURA 22 - Semivariograma experimental da precipitação (mm), do mês de setembro, na Estação Experimental Getúlio Vargas - Uberaba - MG, no período de 1914 a 2000.

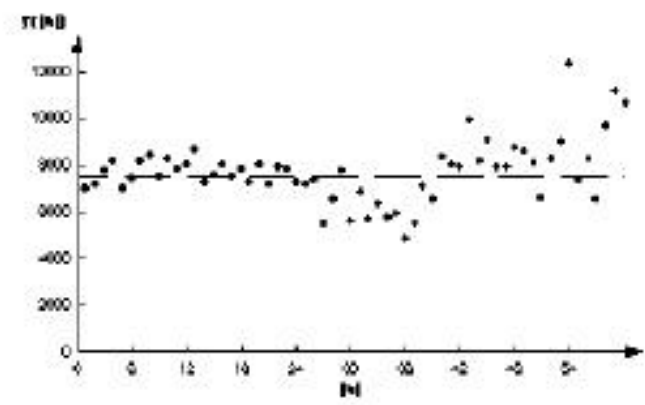

FIGURA 24 - Semivariograma experimental da preci- FIGURA 25 - Semivariograma experimental da precipitação (mm), do mês de novembro, na Estação Expe- pitação (mm), do mês de dezembro, na Estação Experimental Getúlio Vargas - Uberaba - MG, no período rimental Getúlio Vargas - Uberaba - MG, no período de 1914 a 2000.

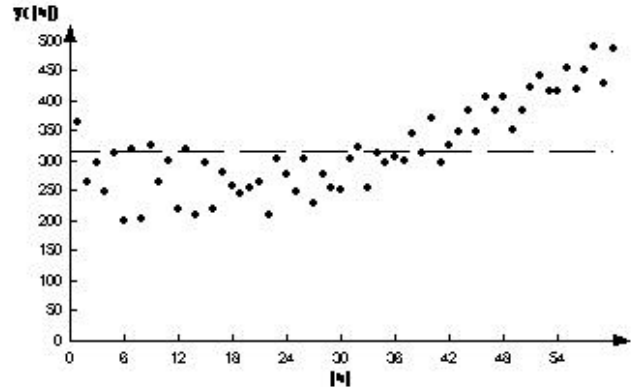

FIGURA 21 - Semivariograma experimental da precipitação (mm), do mês de agosto, na Estação Experimental Getúlio Vargas - Uberaba - MG, no período de 1914 a 2000.

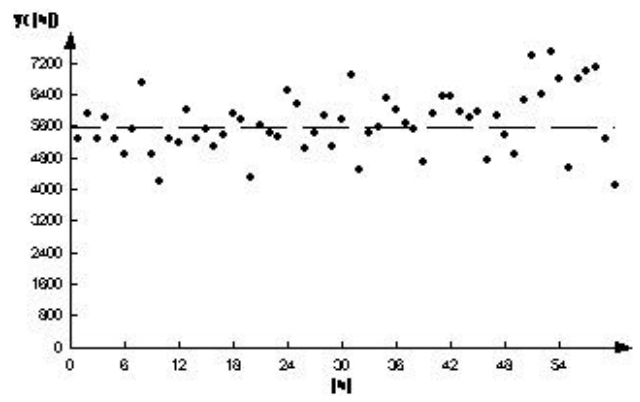

FIGURA 23 - Semivariograma experimental da precipitação (mm), do mês de outubro, na Estação Experimental Getúlio Vargas - Uberaba - MG, no período de 1914 a 2000.

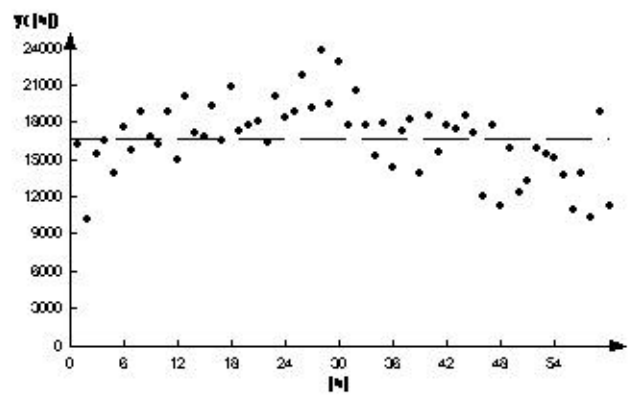
de 1914 a 2000. 


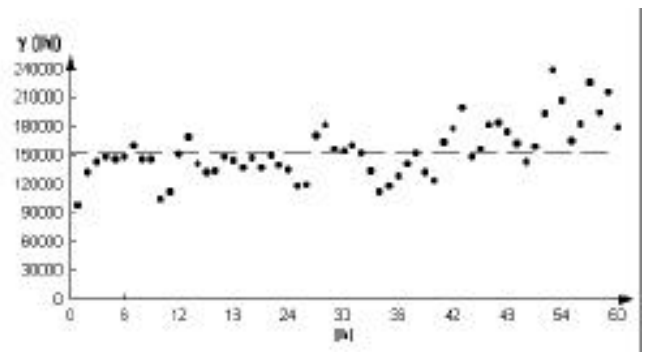

FIGURA 26 - Semivariograma experimental da precipitação anual (mm), na Estação Experimental Getúlio Vargas - Uberaba - MG, no período de 1914 a 2000 .

Fatores que podem ter contribuído com o fato de se ter observado a dependência temporal fraca ou a ausência da dependência temporal são: o espaçamento de tempo entre as observações que correspondem a um ano, a utilização de totais mensais e anuais e a ausência de registros em alguns anos da série. Em concordância com essas observações, Aspiazú et al. (1990) salientam que a falta de registros contínuos é um dos fatores que dificultam as classificações climáticas e Sansigolo (1996) argumenta sobre a necessidade de uma análise detalhada de registros de precipitação para fins de utilização das informações na agricultura.

Em trabalhos nos quais foram observados dados espaciais como Vieira et al. (1991), Gomes (2001) e Cardim (2001), verificou-se dependência espacial para a variável precipitação avaliada por meio de semivariogramas.

\section{CONCLUSÕES}

a) A precipitação mensal e anual, na Estação Climatológica de Uberaba, apresentou dependência temporal fraca ou não apresentou dependência temporal, para a série estudada.

b) Para os casos em que o semivariograma apresentou efeito pepita puro, as estimativas de precipitação pluviométrica podem ser feitas, considerando independência entre épocas de coleta de dados.

\section{REFERÊNCIAS BIBLIOGRÁFICAS}

ASPIAZÚ, C.; RIBEIRO, G. A.; VIANELLO, R. L.; RIBEIRO, J. C.; VALENTE, O. F.; PAULA NETO, F. Análise dos componentes principais aplicada na classificação climática do estado de Minas Gerais: teste metodológico. Revista Árvore, Viçosa, v. 14, n. 1, p. 115,1990 .
CAMBARDELLA, C. A.; MOORMAN, T. B.; NOVAK, J. M., PARKIN, T. B.; KARLEN, D. L.; TURCO, R. F.; KONOPKA, A. E. Field-scale variabillity of soil properties in central Iowa soils. Soil Science Society America Journal, Madison, v. 58, p. 15011511, 1994.

CAMPELO JUNIOR, J. H. Duração, homogeneidade e distribuição espacial das séries de precipitação em Mato Grosso. Revista Brasileira de Agrometeorologia, Santa Maria, v. 1, n. 1, p. 137-140, 1993.

CARDIM, M. Mapeamento do comportamento multivariado das principais variáveis climáticas de interesse agrícola do estado de São Paulo. 2001. 124 f. Tese (Doutorado em Agronomia) - Universidade Estadual Paulista "Júlio de Mesquita Filho", Faculdade de Ciências Agronômicas, Botucatu.

ELTZ, F. L. F.; REICHERT, J. M.; CASSOL, E. A. Período de retorno de chuvas de Santa Maria, RS. Revista Brasileira de Ciência do Solo, Campinas, v. 16, p. 265-269, 1992.

FREITAS, V. A. de. Análise de dados espaciais por meio de semivariogramas. 2000. 30 f. Monografia (Graduação em Matemática) - Universidade Federal de Uberlândia, Uberlândia.

GOMES, B. M. Comportamento espacial do percentil 75 da precipitação decendial do estado de São Paulo. 2001. 101 f. Tese (Doutorado em Agronomia) - Universidade Estadual Paulista "Júlio de Mesquita Filho", Faculdade de Ciências Agronômicas, Botucatu.

GOMES, F. P. Curso de estatística experimental. Piracicaba: Nobel, 2000. 467 p.

GOOVAERTS, P. Geostatistical approaches for incorporating elevation into the spatial interpolation of rainfall. Journal of Hydrology, Amsterdam, v. 228, p. 113-129, 2000.

GUIMARÃES, E. C. Variabilidade espacial de atributos de um latossolo vermelho escuro textura argilosa da região do cerrado, submetido ao plantio direto e ao plantio convencional. 2000. $89 \mathrm{f}$. Tese (Doutorado em Engenharia Agrícola) - Universidade Estadual de Campinas, Faculdade de Engenharia Agrícola, Campinas. 
MEDINA, B. F.; LEITE, J. A. Probabilidades de chuva em Boa Vista - RR. Pesquisa Agropecuária Brasileira, Brasília, v. 19, n. 12, p. 1437-1441, 1984.

RIBEIRO, A. M.; LUNARDI, D. M. C. A precipitação mensal provável para Londrina - PR, através da função gama. Energia na Agricultura, Botucatu, v. 12, n. 4, p. 37-44, 1997.

PANNATIER, Y. Variowin: software for spatial data analysis in 2D. New York: Springer-Verlag, 1996. 90 p.

SANSIGOLO, C. A. Variabilidade interanual da estação chuvosa no estado de São Paulo. Revista Brasileira de Agrometeorologia, Santa Maria, v. 4, n. 1, p. 101-105, 1996.

TERRA. Cidade Virtual. Clima de Uberaba. Disponível em: <http:/www.terra.com.br/cidades/uba/clima. htm>. Acesso em: 23 ago. 2002.
VIEIRA, S. R.; LOMBARDI NETO, F.; BURROWS, I. T. Mapeamento da chuva diária máxima provável para o estado de São Paulo. Revista Brasileira de Ciência do Solo, Campinas, v. 15, n. 1, p. 93-98, 1991.

VIEIRA, S. R. Curso de atualização em conservação do solo: uso de geoestatística. Campinas: IAC, 1995. 71 p. Apostila.

VIEIRA, S. R. Variabilidade espacial de argila, silte e atributos químicos em parcelas experimentais de um latossolo roxo de Campinas (SP). Bragantia, Campinas, v. 56, n. 1, p. 29-38, 1997.

ZAMBOTI, J. L. Mapas pluviométricos médios mensais no verão para o estado do Paraná, utilizando métodos geoestatísticos. 2001. 117 f. Tese (Doutorado em Agronomia) - Universidade Estadual Paulista "Júlio de Mesquita Filho", Faculdade de Ciências Agronômicas, Botucatu. 